\title{
Differential Weighting of Common and Distinctive Components
}

\author{
Ilana Ritov and Itamar Gati \\ Hebrew University \\ Jerusalem, Israel \\ Amos Tversky \\ Stanford University
}

\begin{abstract}
We investigated possible explanations of the finding that the relative weight $(W)$ of common components in similarity judgments is higher for verbal than for pictorial stimuli. A serial presentation of stimulus components had no effect on verbal stimuli; it increased the impact of both common and distinctive components of pictorial stimuli but did not affect their relative weight. On the other hand, $W$ was increased by manipulations that reduced the cohesiveness of composite pictures, such as separating, scrambling, and mixing their components. Furthermore, $W$ was decreased by manipulations that enhanced the cohesiveness of composite verbal stimuli by imposing structure on their components. Verbal and pictorial representations of the same stimuli yielded no systematic differences in $W$.
\end{abstract}

Objects, concepts, and events are normally organized and categorized on the basis of their common and distinctive features. This notion underlies the contrast model (Tversky, 1977) that expresses the similarity of objects in terms of the measures of their unique and shared features. In this model, each object $a$ is represented as a set of features, denoted $A$, and the similarity of $a$ to $b$, in the symmetric case, is given by

$$
g(A \cap B)-f(A-B)-f(B-A),
$$

where $g(A \cap B)$ is the measure of the features shared by $a$ and $b$, and $f(A-B)$ and $f(B-A)$ are the measures of the features that belong to one object but not to the other.

The contrast model suggests a simple procedure for assessing the relative weight of common and distinctive features. Let $s(p, q)$ denote the rated similarity between objects $p$ and $q$ (e.g., schematic faces), and let $x$ be an additive component (e.g., glasses). Under the natural interpretation of the contrast model, adding $x$ to both $p$ and $q$ increases similarity, and this increment provides an estimate of the impact of $x$ as a common component, denoted $C(x)$; that is,

$$
C(x)=s(p x, q x)-s(p, q) .
$$

Adding $x$ to only one object should reduce similarity, and this decrement provides an estimate of the impact of $x$ as a distinctive component, denoted $D(x)$; that is,

$$
D(x)=s(p, q)-s(p x, q) .
$$

Figure 1 depicts three pairs of faces required for the assessment of the impact of glasses as a common component and as a distinctive component. The relative contribution of these

This work was supported in part by Grant 89-0064 from The Air Force Office of Scientific Research to Stanford University.

Correspondence concerning this article should be addressed to Amos Tversky, Department of Psychology, Stanford University, Stanford, California 94305. components is defined by

$$
W(x)=\frac{C(x)}{C(x)+D(x)} .
$$

Although the features that enter into the contrast model may refer to local or to global properties (e.g., size, symmetry), the procedure for assessing $C(x), D(x)$, and $W(x)$ presupposes that $x$ is a separable, additive component that can be appended to either $p$ or $q$. Indeed, in both past and present studies of the relative weight of common and distinctive features, researchers have used composite stimuli (e.g., Gati \& Tversky, 1987) constructed from a master set of separable components. The necessary conditions for the separability and additivity of the critical component, $x$, were discussed and tested by Gati and Tversky (1984).

These authors constructed many sets of verbal stimuli (e.g., descriptions of persons, meals, trips) and pictorial stimuli (e.g., schematic faces, landscapes) and assessed $C$ and $D$ for more than 50 components. The data yielded a consistent, though unexpected, pattern: $C$ was greater than $D$ (i.e., $W>$ .5) for almost all the verbal components, whereas $D$ was greater than $C$ (i.e., $W<.5$ ) for almost all the pictorial components. For example, the increment in similarity produced by adding glasses to a pair of schematic faces was smaller than the decrement in similarity produced by adding glasses to one face only (see Figure 1). On the other hand, the increment produced by adding a common avocation (e.g., a chess player) to the description of two individuals was greater than the decrement produced by adding this feature to one person only. In this article we investigate several hypotheses that could explain the differential weighting of common and distinctive components.

One factor that affects $W$ is the overall level of similarity between the relevant objects. When asked to compare very similar objects (e.g., two photographs of the same person), people tend to take the shared features for granted and to focus on the distinctive features. On the other hand, in the 


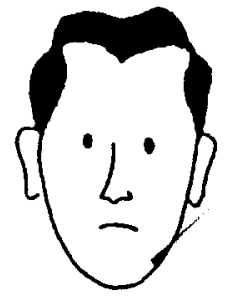

$\mathbf{p}$

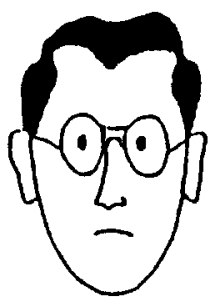

px

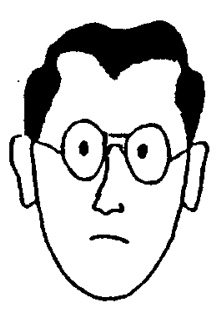

px

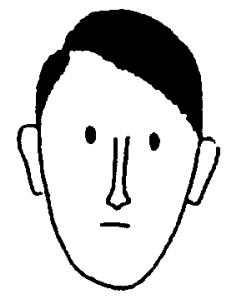

q
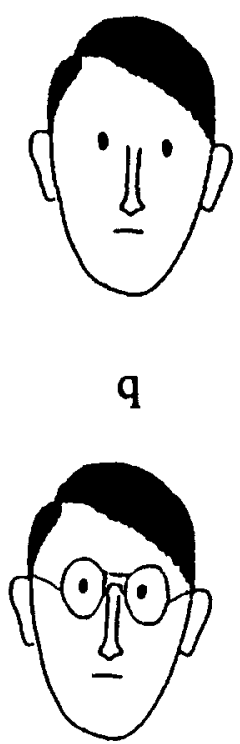

qx
Figure 1. Pairs of faces used to illustrate the assessment of $C$ and D.

comparison of dissimilar objects (e.g., two photographs of a person as a child and as an adult), people tend to take the differences for granted and to focus on the common features. As a consequence, the relative weight of common and distinctive features is inversely related to the overall level of similarity between the objects. Indeed, Gati and Tversky (1984) showed that the contribution of an added common component decreases in the presence of other common components, and the contribution of an added distinctive component decreases in the presence of other distinctive components. This effect may be explained, in part at least, by the subadditivity of the measures $f$ and $g$ in Equation 1. As will be shown later, however, the difference between the verbal and the pictorial stimuli is evident even when similarity is controlled.

The conceptual and the perceptual comparisons that gave rise to the differential weighting of $C$ and $D$ differ not only in the modality (verbal vs. pictorial) but also in other respects that may explain the variations in $W$. Two such differences come to mind. First, it seems that the processing of the verbal stimuli is more serial in nature than that of the pictorial stimuli. Second, it can be argued that the pictorial stimuli used in our studies are more cohesive than the verbal stimuli. In the following series of studies, we investigated these notions. First, we tested the hypothesis that a serial presentation of the stimuli yields higher values of $W$ than does a simultaneous presentation. Next, we investigated the hypothesis that $W$ is inversely related to the cohesiveness of the stimuli. Last, we tested whether verbal and pictorial representations of the same set of objects give rise to systematic differences in $W$.

\section{Serial Processing}

The difference between pictorial and verbal comparisons, with respect to $W$, may be produced by the different modes of processing. Verbal stimuli that consist of lists of items are bound to be processed serially. The pictorial stimuli, on the other hand, do not impose a fixed linear order and may be processed in a more parallel fashion. The differential weighting effect, therefore, could be explained by the hypothesis that
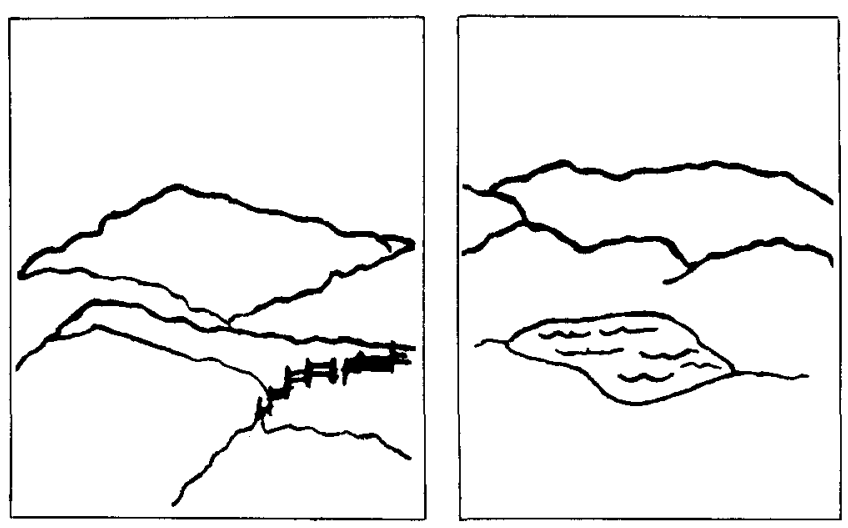

p
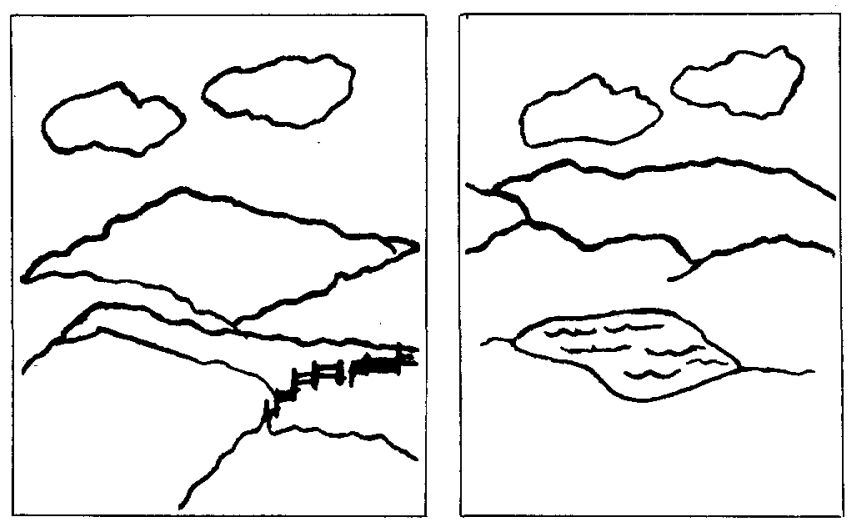

px

qX

Figure 2. An illustration of serial presentation from Design I. 


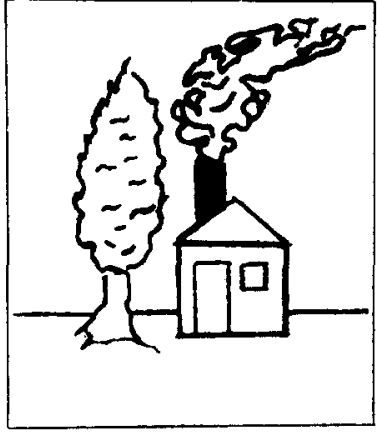

pxy

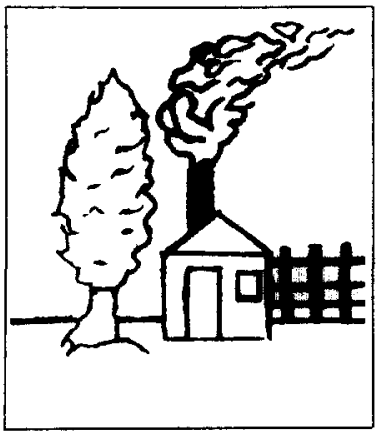

pxyz
Figure 3. A pair of stimuli from Design II.

serial processing emphasizes common features, whereas parallel processing emphasizes distinctive features.

\section{Experiment 1}

We tested this hypothesis by presenting both verbal and pictorial stimuli either simultaneously or serially. In the simultaneous condition, all components were presented at once. In the serial condition, subjects were first presented with the basic pair of stimuli $(p, q)$, and the critical component $(x)$ was added after $2 \mathrm{~s}$. Subjects were asked to judge the similarity between the composite stimuli $(p x, q x)$ after the presentation of all the components. An example of the serial presentation of the pictorial stimuli is presented in Figure 2. Both verbal and pictorial stimuli were displayed on a monitor.

If the modality effect is caused by the serial processing of the verbal stimuli, then we would expect little or no difference between the simultaneous and the serial conditions for the verbal stimuli and a substantial difference between the conditions for the pictorial stimuli. Thus we expected lower $W$ in the pictorial-simultaneous condition than in the pictorialserial condition.

\section{Method}

Subjects. The subjects in all of the following experiments were undergraduate students at the Hebrew University in Jerusalem. Some participated to fulfill course requirements; others were paid for participation. Subjects were randomly assigned to the experimental conditions.

Stimuli. Two families of pictorial components and two families of verbal components were used for the construction of the stimuli. Two pairs of stimuli from the first family are presented in Figure 2, and a pair of stimuli from the second family is presented in Figure 3. The verbal stimuli consisted of descriptions of individuals in terms of their occupation, political affiliation, avocation, and character. All stimulus components are listed in Table 1.

Design. Two designs for stimulus construction were used in the study. In Design I, each stimulus consisted of one of two landscapes ( $p$ or $q$ ), with $x$ and $y$ as the additive components. The following six pairs of stimuli were used in this design: $(p, q),(p x, q x),(p, p x)$, $(p x, p y),(p y, p)$, and $(q y, p y)$. In Design II, each stimulus includes a house ( $p$ ), with $x, y$, and $z$ as additive components. The following six pairs of stimuli were used in this design: $(p y z, p x y),(p x, p x z),(p y z, p y)$, $(p x, p z),(p x y, p x z)$, and $(p y, p z)$. The designs of the verbal stimuli followed those of the pictorial stimuli (see Table 1).

Forty-four subjects were assigned to the simultaneous condition, and the same number of subjects were assigned to the serial condition. Each subject was presented with all six pairs of stimuli from each of the four families. The pairs of each family were grouped together and presented in the same random orders in both conditions. The pictorial and the verbal families appeared in an alternating random order.

Procedure. The experiment was controlled by an Apple IIE computer, and the stimuli were presented on a TAXAN RGB color monitor. Subjects were presented with pairs of stimuli and were asked to rate the similarity between them on a scale from 1 (low similarity) to 20 (high similarity), using a numerical keyboard. Subjects were tested individually, and the experiment lasted about $20 \mathrm{~min}$.

\section{Results}

For each additive component, $x$, we computed $C(x)$ and $D(x)$ separately for each subject, following the procedure developed by Gati and Tversky (1984):

$$
\begin{array}{ll}
\text { Design I: } & C(x)=s(p x, q x)-s(p, q) \quad \text { and } \\
& D(x)=s(p, p y)-s(p x, p y) ; \\
\text { Design II: } & C(x)=s(p x y, p x z)-s(p y, p z), \quad \text { and } \\
& D(x)=s(p y z, p y)-s(p y z, p y x) .
\end{array}
$$

Recall that $C(x)$ and $D(x)$ are measures of, respectively, the

\begin{tabular}{|c|c|c|c|c|}
\hline Item & Family 1 & Family 2 & Family 3 & Family 4 \\
\hline $\begin{array}{l}\text { Design I } \\
\text { Stimuli } \\
\text { Verbal }\end{array}$ & $\begin{array}{c}\text { p } \\
\text { High school } \\
\text { teacher }\end{array}$ & $\begin{array}{c}\mathbf{q} \\
\text { Tax consultant }\end{array}$ & $\begin{array}{c}\mathbf{x} \\
\text { Naturalist }\end{array}$ & $\stackrel{\mathrm{y}}{\text { Socialist }}$ \\
\hline $\begin{array}{l}\text { Pictorial } \\
\text { Design II }\end{array}$ & Mountains & Lake & Cloud & House \\
\hline $\begin{array}{l}\text { Stimuli } \\
\text { Verbal }\end{array}$ & $\begin{array}{c}\mathrm{p} \\
\text { Student }\end{array}$ & $\frac{z}{\substack{\text { Human-rights } \\
\text { activist }}}$ & $\begin{array}{l}x \\
\text { Soccer } \\
\text { fan }\end{array}$ & $\begin{array}{c}y \\
\text { Arrogant }\end{array}$ \\
\hline Pictorial & House & Fence & Tree & Smoke in chimney \\
\hline
\end{tabular}
contributions of $x$ as a common component and as a distinc-

Table 1

Four Families of Stimuli Used in Experiment 1 
tive component. We also computed their difference, $\delta(x)=$ $C(x)-D(x)$, and their normalized ratio, $W(x)=C(x) / C(x)+$ $D(x)$, which reflect the relative impact of $C$ and $D . W(x)>.5$ if and only if $\delta(x)>0$. Because $W$ is a measure of the relative contribution of $C$ and $D$, it is generally independent of the salience of the estimated feature; hence it provides a useful descriptive index. For the purpose of hypothesis testing, however, we always use the difference $\delta$, instead of the ratio $W$, because it is linear in $C$ and $D$.

We computed the mean estimates, across subjects, of $C, D$, $\delta$, and $W$ for each of the additive components in both the simultaneous and the serial conditions (see Table 2). We also computed, for each subject, the average value of these measures separately for the verbal and for the pictorial components (see Table 3). Because $W$ is a ratio, we report its median, rather than the arithmetic mean.

Table 2 shows that $W<.5$ for all pictorial components and $W>.5$ for all verbal components in both the simultaneous and the serial conditions. The within-subject analysis (see Table 3) revealed the same pattern. For the verbal stimuli, the mode of presentation (simultaneous vs. serial) had no effect on any of the four indices $(C, D, \delta$, and $W)$. A two-way analysis of variance (ANOVA) of the verbal data showed that

Table 2

Data From Simultaneous and Serial Presentations for Each Additive Component

\begin{tabular}{|c|c|c|c|c|}
\hline Additive component & $C$ & $D$ & $\delta$ & $W$ \\
\hline \multicolumn{5}{|c|}{ Verbal stimuli } \\
\hline \multicolumn{5}{|l|}{ Family 1: Naturalist } \\
\hline Simultaneous & 6.65 & 0.34 & 6.31 & .95 \\
\hline Serial & 7.16 & 0.55 & 6.60 & .92 \\
\hline \multicolumn{5}{|l|}{ Family 1: Socialist } \\
\hline Simultaneous & 7.36 & -0.22 & 7.58 & $1.03^{\mathrm{a}}$ \\
\hline Serial & 7.32 & 0.95 & 6.37 & .88 \\
\hline \multicolumn{5}{|l|}{$\begin{array}{l}\text { Family 2: Soccer } \\
\text { fan }\end{array}$} \\
\hline Simultaneous & 5.75 & 0.52 & 5.22 & .91 \\
\hline Serial & 5.27 & 1.39 & 3.88 & .79 \\
\hline \multicolumn{5}{|l|}{ Family 2: Arrogant } \\
\hline Simultaneous & 6.22 & 0.45 & 5.77 & .93 \\
\hline Serial & 5.83 & 0.44 & 5.39 & .92 \\
\hline
\end{tabular}

\begin{tabular}{lllll} 
Family 3: Cloud & & & & \\
$\quad$ Simultaneous & 0.63 & 1.40 & -0.77 & .31 \\
$\quad$ Serial & 1.55 & 2.76 & -1.20 & .35 \\
Family 3: House & & & & \\
$\quad$ Simultaneous & 1.43 & 4.09 & -2.65 & .25 \\
$\quad$ Serial & 2.25 & 4.27 & -2.02 & .34 \\
$\quad$ Family 4: Tree & & & & \\
$\quad$ Simultaneous & 0.93 & 2.68 & -1.75 & .25 \\
$\quad$ Serial & 2.53 & 4.79 & -2.25 & .34 \\
Family 4: Smoke & & & & \\
$\quad$ Simultaneous & 1.27 & 2.70 & -1.43 & .31 \\
$\quad$ Serial & 1.76 & 4.30 & -2.53 & .29 \\
\hline
\end{tabular}

Note. $C=$ weight of a feature as a common component. $D=$ weight of a feature as a distinctive component. $\delta=C-D . W=C /(C+D)$. " This value of $W$ exceeds 1.00 because $D$ was negative, although it was not significantly different from 0 .
Table 3

Summary Results for Experiment 1 (Simultaneous and Serial Presentation)

\begin{tabular}{lllll}
\hline Stimuli & $C$ & $D$ & $\delta$ & $W$ \\
\hline Verbal & & & & \\
Simultaneous & 6.50 & 0.27 & 6.22 & .94 \\
$\quad$ Serial & 6.40 & 0.83 & 5.56 & .93 \\
Pictorial & & & & \\
$\quad$ Simultaneous & 1.06 & 2.72 & -1.65 & .26 \\
$\quad$ Serial & 2.02 & 4.03 & -2.00 & .33 \\
\hline
\end{tabular}

Note. $C=$ weight of a feature as a common component. $D=$ weight of a feature as a distinctive component. $\delta=C-D . W=C /(C+D)$.

$C$ was significantly larger than $D, F(1,85)=240.89, M S_{\mathrm{e}}=$ $6.27, p<.01$, but the presentation mode had no effect, $F(1$, $85)=0.32, M S_{\mathrm{e}}=7.34, n s$. The interaction was also not significant, $F(1,85)=0.76, M S_{\mathrm{e}}=6.27$.

The results for the pictorial stimuli are quite different: $D$ was significantly greater than $C, F(1,85)=52.61, M S_{\mathrm{e}}=2.76$, $p<.01$, and the estimates of both $C$ and $D$ were higher in the serial condition than in the simultaneous condition, $F(1,85)=14.20, M S_{\mathrm{e}}=3.95, p<.01$. The interaction between these factors was not significant, $F(1,85)=0.48$, $M S_{\mathrm{e}}=2.76$.

\section{Discussion}

The results of Experiment 1 may be summarized as follows. First, we have replicated the major finding of Gati and Tversky (1984): namely, that for verbal stimuli, $C$ exceeds $D$, and hence $W>.5$, whereas for pictorial stimuli, $D$ exceeds $C$, and hence $W<.5$. Second, the serial presentation of stimulus components had no effect on the verbal stimuli, presumably because they are processed serially even in the simultaneous condition. Third, the serial presentation of the pictorial stimuli enhanced the impact of the added common and distinctive components but did not increase $W$. Evidently, the differential weighting of common and distinctive components cannot be explained by the serial processing of the verbal stimuli.

\section{Reducing the Cohesiveness of Pictorial Stimuli}

Another possible explanation of the differential weighting effect is that the pictorial stimuli used in previous studies (e.g., line drawings of landscapes or schematic faces) were more cohesive than the verbal stimuli with which they were compared (e.g., descriptions of people, meals, or trips). Because the pictorial stimuli represent three-dimensional objects and scenes, their components must satisfy certain mutual constraints regarding position, relative size, continuity, and so on, which render them more cohesive. In contrast, the verbal stimuli appear less cohesive because they consist of items with few or no mutual constraints. According to this account, reducing the cohesiveness of the pictorial stimuli should increase $W$, and enhancing the cohesiveness of the verbal stimuli should decrease $W$. Although we do not have a general 
procedure for measuring the cohesiveness of pictorial or of verbal stimuli, we can identify several manipulations, or transformations, that are assumed to increase or reduce the cohesiveness of objects. In the next six studies, we applied these transformations to pictorial stimuli (Experiments 2, 3, 4, and 5) and to verbal stimuli (Experiments 6 and 7) and investigated their impact on $C, D$, and $W$.

\section{Experiment 2}

In this experiment we manipulated the degree to which the components of a stimulus appear as separate parts or as a coherent picture. The stimuli were line drawings composed of two, three, or four components displayed in the same positions. In Condition 1, a horizontal line appeared in the background, creating an impression of a natural scene. In Condition 2, the horizontal line was deleted. In Condition 3, the same components were separated by vertical lines. Figures 4 and 5 are examples of two families of components displayed under the three conditions. If the cohesiveness of the picture were inversely related to $W$, we would expect the highest $W$ in Condition 3 and the lowest $W$ in Condition 1.

\section{Method}

Stimuli. Ten families of components were used to construct the stimuli. The following three pairs were constructed for each family: $(p, q),(p x, q x)$, and $(p x, q)$. The stimulus $p$ consisted of three items, whereas $q$ included two items, one of which was also included in $p$. The additive component $x$ was not included in either $p$ or $q$.

Design and procedure. In this experiment, as in all the following studies, the stimuli were presented in booklets, and the members of each pair were displayed side by side. Subjects were asked to rate the similarity of each pair on a scale from 1 (low similarity) to 20 (high similarity). They were tested in a classroom setting in groups of 3090. The subjects received written instructions and an example on the first page of the booklet. In this experiment, 83 students were randomly assigned to one of three experimental conditions. Each subject rated all 30 pairs of stimuli. The order of the pairs was randomized across sets, and the same ordering were used in all three conditions. The experiment lasted $15 \mathrm{~min}$.

\section{Results}

For each of the ten additive components, we calculated the values of $C$ and $D$ separately for each subject, following

\section{Horizontal line}
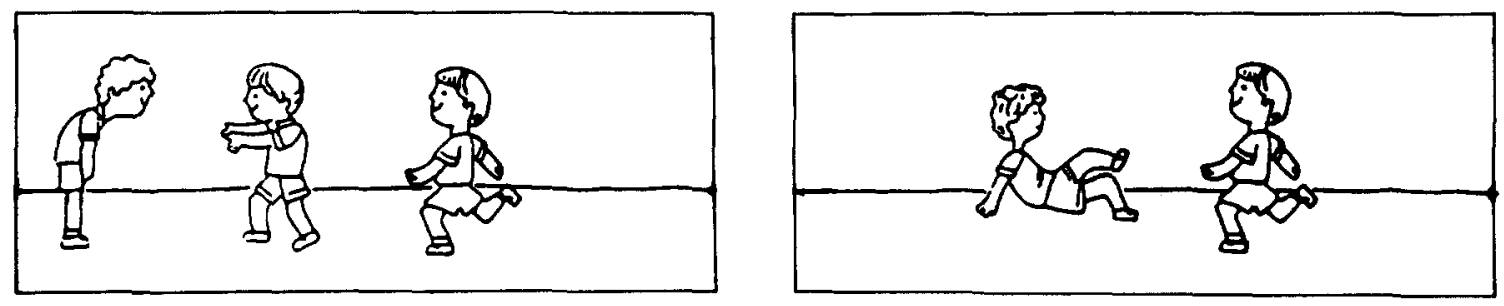

No line
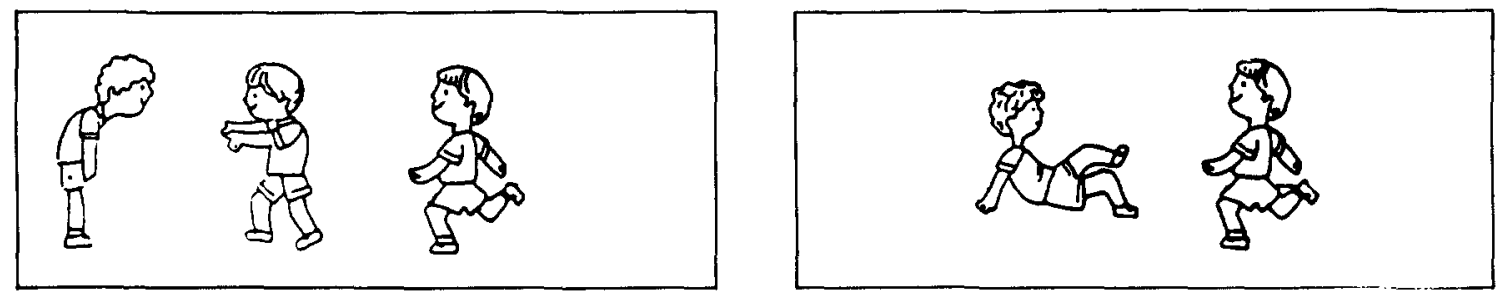

\section{Vertical} line
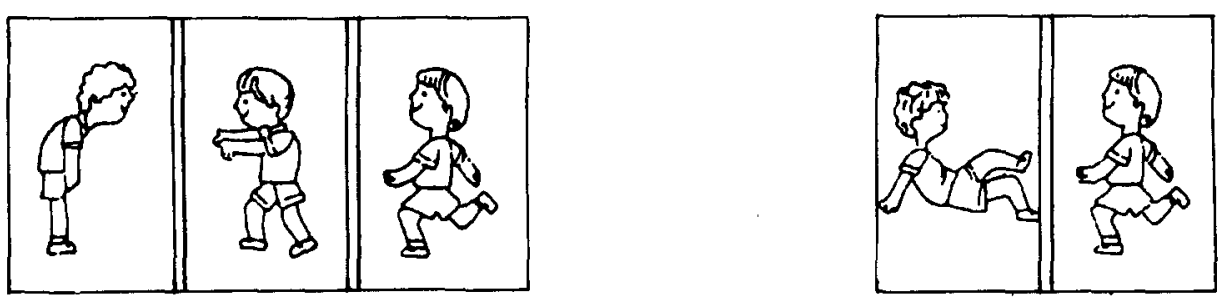

Figure 4. An example of the displays used in Experiment 2. 

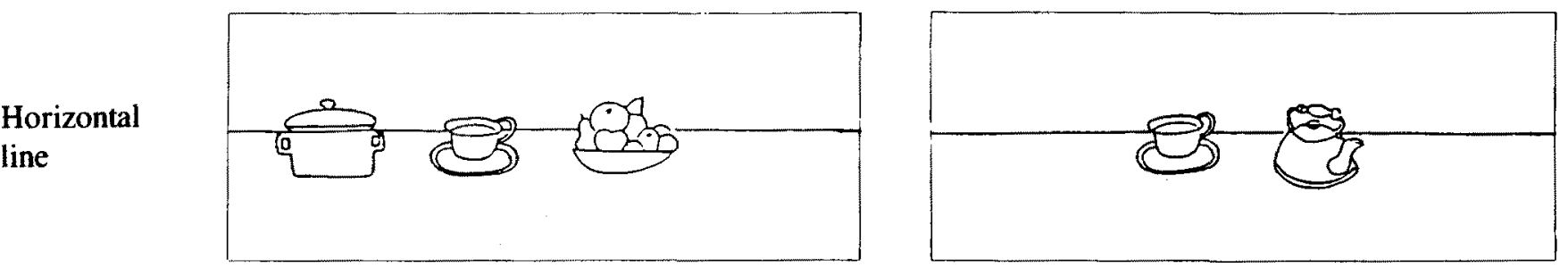

No line
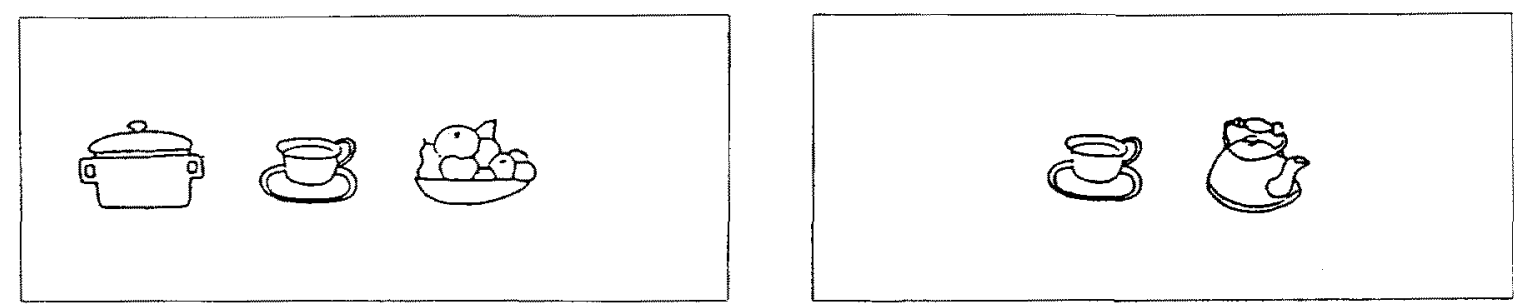

Vertical
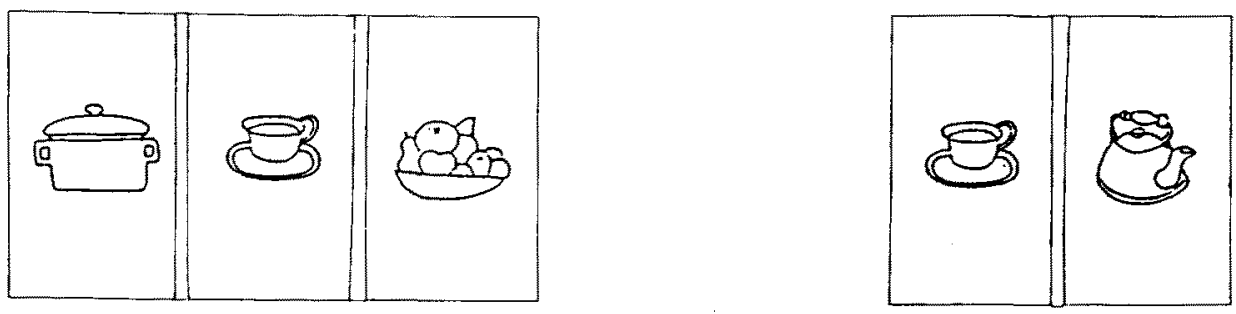

Figure 5. Another example of the pictorial stimuli used in Experiment 2.

Equations 2 and 3 . The mean values of $C, D$, and $\delta$ and the median $W$ are presented in Table 4.

The table shows that both $W$ and $\delta$ are lowest in the presence of the horizontal lines, which underscore the cohesiveness of the pictures, and highest in the presence of the vertical lines. A one-way ANOVA yielded a statistically significant difference in $\delta$ among the three conditions, $F(2,80)=3.65, M S_{\mathrm{e}}=2.76$, $p<.05$. It is noteworthy that the effect of the horizontal line is significant, whereas the effect of the vertical line is not. This observation suggests that in the absence of a line, the picture is perceived as a collection of components, not as an integrated scene. Also, $W>.5$ in all three conditions, indicating that the common components loom larger than the distinctive components in the composite pictures, unlike the scenes from Experiment 1.

\section{Experiment 3}

In this study, we manipulated the cohesiveness of natural scenes by scrambling their components. Examples of natural and scrambled scenes are presented in Figure 6 . If $W$ were affected by the cohesiveness of the stimuli, we would expect higher $W$ in the scrambled pictures than in the natural pictures.

\section{Method}

Five families of components were used: flatware, office furniture, a living room, a street scene, and an airport. For each family, we constructed six pairs of stimuli of the forms $(p x, p z),(p y, p z)$, $(p x, p x z),(p x y, p x z),(p y, p y z)$, and $(p x y, p y z)$. The same random orders of all 30 pairs were used in the two conditions. One group of subjects $(n=41)$ rated the similarity of the natural scenes, and the other group of subjects $(n=50)$ rated the similarity of the scrambled scenes.

\section{Results}

The data summarized in Table 5 support the hypothesis that the scrambled condition produced higher values of $W$ and of $\delta, t(89)=1.70, p<.05$, one-tailed.

Table 4

Summary Results for Experiment 2

\begin{tabular}{lccccc}
\hline Condition & $n$ & $C$ & $D$ & $\delta$ & $W$ \\
\hline Horizontal line & 28 & 2.51 & 1.08 & 1.42 & .73 \\
No line & 27 & 3.09 & 0.59 & 2.49 & .81 \\
Vertical line & 28 & 3.20 & 0.54 & 2.65 & .82 \\
\hline
\end{tabular}

Note. $C=$ weight of a feature as a common component. $D=$ weight of a feature as a distinctive component. $\delta=C-D . W=C /(C+D)$. 


\section{Natural}
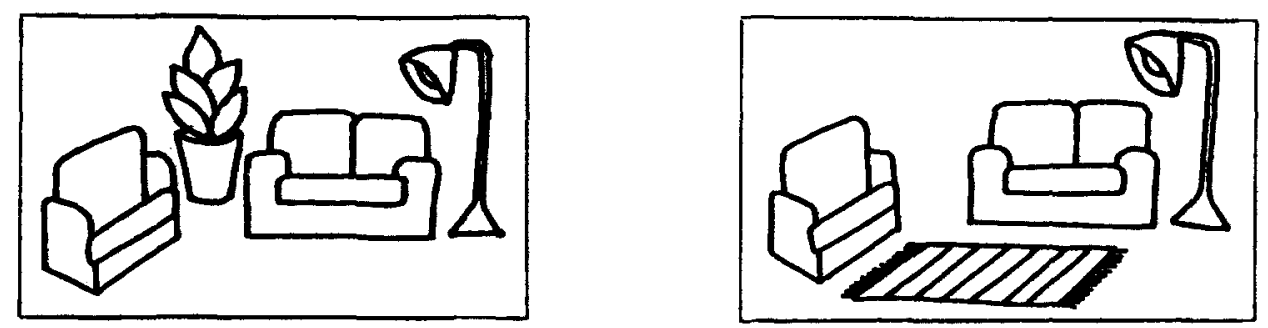

\section{Scrambled}
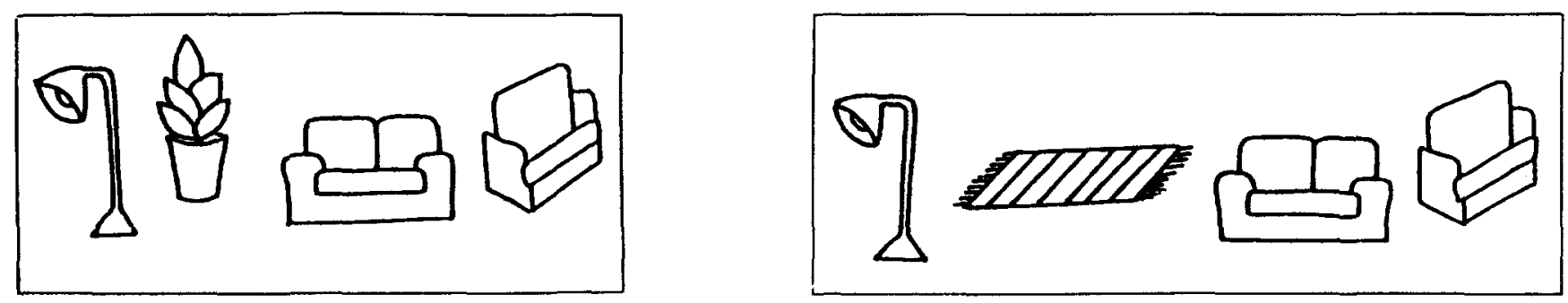

Figure 6. An example of the scrambling manipulation used in Experiment 3.

\section{Experiment 4}

The stimuli in this experiment consisted of series of pictures separated by vertical lines, as in Experiment 2 . We constructed two types of stimuli: uniform and mixed. The components of a uniform stimulus were consistent with respect to content and scale. We constructed the mixed stimuli by mixing the components of two uniform stimuli. Examples of uniform and mixed pairs are presented in Figure 7. Because the mixed stimuli were less cohesive than the uniform ones, we expected higher $W$ for the mixed pictures.

\section{Method}

Six families of components were used: a playground, a building, children, adults, caged animals, and dogs. We obtained the mixed families by mixing adjacent pairs of families. Three pairs of stimuli, as in Experiment 2, were constructed for each family. One group of subjects $(n=28)$ rated the similarity of 18 pairs of uniform stimuli, and another group of subjects $(n=31)$ rated the similarity of all 18 pairs of mixed stimuli.

\section{Results}

The data summarized in Table 6 show that $C$ was higher in the mixed condition than in the uniform condition, whereas $D$ was higher in the uniform condition than in the mixed condition. As a consequence, $W$ and $\delta$ were greater in the mixed condition than in the uniform condition, $t(57)=$ $5.00, p<.001$, in accordance with the cohesiveness hypothesis.

\section{Experiment 5}

The target stimuli for this experiment were composite pictures displayed with vertical lines, taken from Experiment 2. Target stimuli were presented with other stimuli-uniform or mixed-taken from Experiment 4. If the target stimuli appeared less cohesive in the mixed context than in the uniform context, then we would expect smaller $W$ and $\delta$ in the latter condition than in the former.

\section{Method}

The first five families of components from Experiment 2 were used as target stimuli. For each target family, we constructed three pairs of stimuli, as in Experiment 2 . The three pairs from each family appeared consecutively, alternating with the context families. The context stimuli consisted of the six families from Experiment 4 . One group of subjects $(n=28)$ were presented with the uniform context, whereas another group of subjects $(n=31)$ were presented with the mixed context. The data for Experiments 4 and 5 were collected concurrently.

Table 5

Summary Results for Experiment 3

\begin{tabular}{lccccc}
\hline Condition & $n$ & $C$ & $D$ & $\delta$ & $W$ \\
\hline Natural & 41 & 1.09 & 0.99 & 0.10 & .51 \\
Scrambled & 50 & 0.84 & 0.02 & 0.83 & .58 \\
\hline
\end{tabular}

Note. $C=$ weight of a feature as a common component. $D=$ weigH of a feature as a distinctive component. $\delta=C-D . W=C /(C+D$. 
Uniform
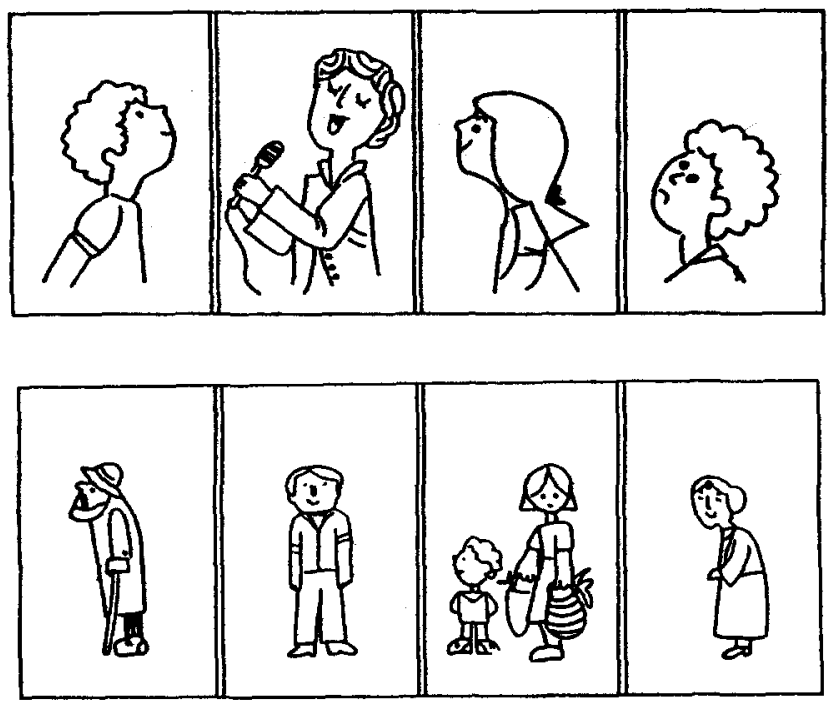

Mixed

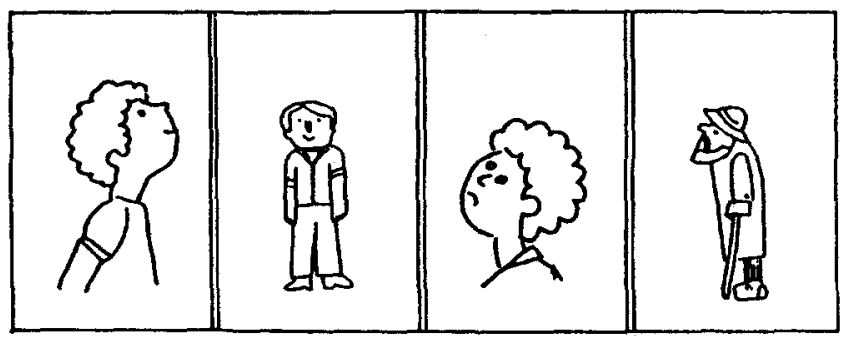

Figure 7. An example of the mixing manipulation used in Experiment 4.

\section{Results}

The data summarized in Table 7 show that, as in the previous study, $C$ was greater in the mixed context than in the uniform context, whereas $D$ was greater in the uniform context than in the mixed context. As expected, $W$ and $\delta$ were higher in the mixed condition than in the uniform condition, $t(57)=5.83, p<.001$.

\section{Imposing Structure on Verbal Stimuli}

In the preceding experiments, we tested the effects of manipulations that perturb the structure of pictorial stimuli. In the following two studies, we investigated the effect of manipulations designed to enhance the cohesiveness of verbal stimuli.

\section{Experiment 6}

The stimuli in this experiment were descriptions of agricultural farms. In the classified condition, each farm was de-
Table 6

Summary Results for Experiment 4

\begin{tabular}{lccccc}
\hline Condition & $n$ & $C$ & $D$ & $\delta$ & $W$ \\
\hline Uniform & 28 & 2.10 & 1.80 & 0.30 & .57 \\
Mixed & 31 & 3.70 & 0.94 & 2.75 & .79 \\
\hline
\end{tabular}

Note. $C=$ weight of a feature as a common component. $D=$ weight of a feature as a distinctive component. $\delta=C-D . W=C /(C+D)$.

scribed in terms of its branches (e.g., peanuts, olives, and poultry), each subsumed under the appropriate category (crops, fruits, and livestock). An example of a pair of farms, as presented in the classified condition, is displayed in Table 8. In the unclassified condition, the same components were presented without the defining labels. Because the classification of the components imposed an additional structure on the stimuli, we expected lower $W$ in the classified condition than in the unclassified condition.

\section{Method}

Six families of components were used. For each family, we constructed three pairs as in Experiment 2. Each farm included no more than one item from each of the four categories. The additive component, $x$, was livestock (e.g., poultry, fish, or sheep). The basic pair $(p, q)$ was constructed so that $p$ included three items and $q$ included two. Forty-five subjects rated the similarity of the farms in the classified condition, and 50 subjects rated the similarity of the same farms in the unclassified condition. The 18 pairs of farms appeared in the same random orders in both conditions.

\section{Results}

The data summarized in Table 9 show that the classified descriptions produced higher values of $W$ and of $\delta$, as hypothesized, although the difference in $\delta$ was not significant.

\section{Experiment 7}

The stimuli in this experiment were two types of meals served at a student cafeteria: dinners and snacks. A typical dinner consisted of an appetizer, a main course, and a dessert (e.g., pea soup, barbequed chicken with rice, and apple strudel). The snacks were composed of assorted items (e.g., egg salad sandwich, french fries, and cola). Assuming that the

Table 7

Summary Results for Experiment 5

\begin{tabular}{lccccc}
\hline Context & $n$ & $C$ & $D$ & $\delta$ & $W$ \\
\hline Uniform & 28 & 1.98 & 1.56 & 0.41 & .54 \\
Mixed & 31 & 3.83 & 0.63 & 3.19 & .86 \\
\hline
\end{tabular}

Note. $C=$ weight of a feature as a common component. $D=$ weight of a feature as a distinctive component. $\delta=C-D . W=C /(C+D)$. 
Table 8

Example of a Pair of Farms: Experiment 6

\begin{tabular}{ccclc}
\hline Farm & Field crops & Fruits & Vegetables & Livestock \\
\hline A & Wheat & Apricots & Onions & Sheep \\
B & Barley & - & Tomatoes & Sheep \\
\hline
\end{tabular}

dinners are more structured than the snacks, we expected lower $W$ for dinners than for snacks.

\section{Method}

The design was identical to that of Experiment 6. Each dinner included a main course and either a dessert or an appetizer. The missing course served as an additive component. The snacks were composed of two or three items. The same set of additive components was used for the dinners and for the snacks. One group of 33 subjects rated the similarity between the dinners, and another group of 38 subjects rated the similarity between the snacks.

\section{Results}

The data summarized in Table 10 show that $C$ was larger for snacks than for dinners, whereas $D$ was larger for dinners than for snacks. As a consequence, $W$ and $\delta$ were higher for snacks than for dinners, $t(69)=4.26, p<.01$. These results indicate that $W$ was lower in structured stimuli (e.g., dinners and classified farms) than in unstructured stimuli (e.g., snacks and unclassified farms).

\section{Isomorphic Representations}

In the next two experiments, we used stimuli that can be represented either pictorially or verbally. If visual comparisons produced lower $W$ than conceptual comparisons, we would expect a difference between the pictorial and the verbal representations. If, on the other hand, the observed variations in $W$ were governed by factors other than the modality, no systematic difference would be expected.

\section{Experiment 8}

The stimuli in this experiment were achievement profiles of students in several college courses. Performance in each course was described in terms of three levels: high, average, and low. Each achievement profile was presented either graphically as a histogram or as a list. Examples of pairs of profiles displayed as histograms and lists are presented in Figure 8 and Table 11 , respectively.

Table 9

Summary Results for Experiment 6 (Farms)

\begin{tabular}{lrcccc}
\hline Condition & $n$ & $C$ & $D$ & $\delta$ & $W$ \\
\hline Classified & 93 & 2.39 & 1.71 & 0.67 & .64 \\
Unclassified & 111 & 2.72 & 1.77 & 0.95 & .67 \\
\hline
\end{tabular}

Note. $C=$ weight of a feature as a common component. $D=$ weight of a feature as a distinctive component. $\delta=C-D . W=C /(C+D)$.

\section{Method}

Four families of components were used in this study. For each family, we constructed three pairs of profiles: $(p, q),(p x, q x)$, and $\left(p y_{1}, q y_{2}\right)$ (see Figure 8). In this design, the subject always compares profiles with an equal number of components. Each of the 90 subjects was presented with two families in tabular form and with two families in graphic form. Half of the subjects evaluated the pictorial stimuli first and the verbal stimuli later; the other half of the subjects performed these tasks in the opposite order. They were asked to rate the similarity between the academic achievement of students.

\section{Results}

Because the distinctive features in this study are substitutive rather than additive, we had to use a different procedure for estimating their impact. Specifically, we defined $D(x)$ as follows:

$$
D^{\prime}(x)=s(p, q)-s\left(p y_{1}, p y_{2}\right) .
$$

The within-subject data summarized in Table 12 show that there were no differences between the graphs and the lists with respect to all four indices, $F(1,89)=0.09, M S_{\mathrm{e}}=16.26$, $n s$, for the difference in $\delta . W<.5$ in both conditions. These results suggest that for highly structured stimuli, there might be no systematic difference in $W$ between pictorial and verbal representations.

\section{Experiment 9}

The stimuli in this experiment were compound geometric figures (e.g., a triangle circumscribed by a circle or a pentagon with a horizontal line connecting two vertices). Because such stimuli can be fully characterized verbally, as well as pictorially, they can be used to test the effect of modality on $\delta$ and $W$.

\section{Method}

Three families of components were used. Three pairs of stimuli were constructed from each family, in accordance with the design used for landscapes in Experiment 1. One group of subjects $(n=25)$ rated the similarity between the 18 pairs of figures, whereas another group $(n=22)$ rated the similarity between their verbal descriptions.

\section{Results}

The results summarized in Table 13 show that there were no significant differences, $t(45)=-0.82, n s$, between the verbal and the pictorial descriptions of the geometric figures.

\section{General Discussion}

In this series of studies, we explored several hypotheses regarding the determinants of the relative weight of common and distinctive components. In Experiment 1 we showed that the serial presentation of stimulus components, designed to encourage serial processing, had no effect on verbal stimuli. 
Table 10

Summary Results for Experiment 7 (Meals)

\begin{tabular}{cccccc}
\hline Condition & $n$ & $C$ & $D$ & $\delta$ & $W$ \\
\hline Dinners & 33 & 1.76 & 2.95 & -1.19 & .26 \\
Snacks & 38 & 3.59 & 1.32 & 2.27 & .77 \\
\hline
\end{tabular}

Note. $C=$ weight of a feature as a common component. $D=$ weight of a feature as a distinctive component. $\delta=C-D, W=C /(C+D)$.

For the pictorial stimuli, the serial presentation increased the impact of both common and distinctive components, but it did not change their relative weight. These results indicate that the observed difference in $W$ between the verbal and the pictorial stimuli cannot be attributed to the serial processing of the verbal stimuli. In Experiments 8 and 9, we showed no significant difference in $W$ between verbal and pictorial representations of the same stimuli, which indicates that variations in $W$ cannot be attributable to modality alone.

Our main finding concerns the effect of cohesiveness. In the absence of a general procedure for measuring cohesiveness, we attempted to manipulate this variable in several experiments. In Experiments 2, 3, 4, and 5, we showed that $W$ was decreased by manipulations that reduced the cohesiveness of composite pictures by separating, scrambling, and mixing their components. In Experiments 6 and 7, we showed that $W$ was increased by manipulations that enhanced the cohesiveness of verbal stimuli by imposing structure on the components. In this section, we discuss some alternative interpretations of this effect.
A possible explanation of the variations in $W$ and of the effect of cohesiveness involves the overall level of similarity between the stimuli. Gati and Tversky (1984) showed that the value of $W$ associated with a particular component, added to one or two objects, is inversely related to the similarity of the objects. In particular, the impact of a common or distinctive component decreases in the presence of, respectively, other common or distinctive components. In general, it appears that the comparison of very similar objects calls attention to their distinctive features, and the comparison of dissimilar objects calls attention to their common features. Because cohesiveness is a significant feature of objects, it is possible that the cohesive pairs are more similar to each other than are the noncohesive pairs. Hence the inverse relation between cohesiveness and $W$ may be caused by the greater similarity of the cohesive pairs. To test this hypothesis, we compared the mean baseline similarity, across all pairs of stimuli that serve as a basis for the additive components, in the cohesive and the noncohesive conditions. This analysis was applied to all the experiments in which essentially the same stimuli appeared in the cohesive and the noncohesive conditions. The results are summarized in Table 14, in which we also present the corresponding values of $\delta$ for both conditions.

The table shows that, contrary to the hypothesis just presented, baseline similarity was actually lower in the cohesive condition than in the noncohesive condition in five of seven studies. The difference in baseline similarity was significant only in Experiment 6, but in this study there was no significant

\section{CHEMISTRY}

\section{Student I}

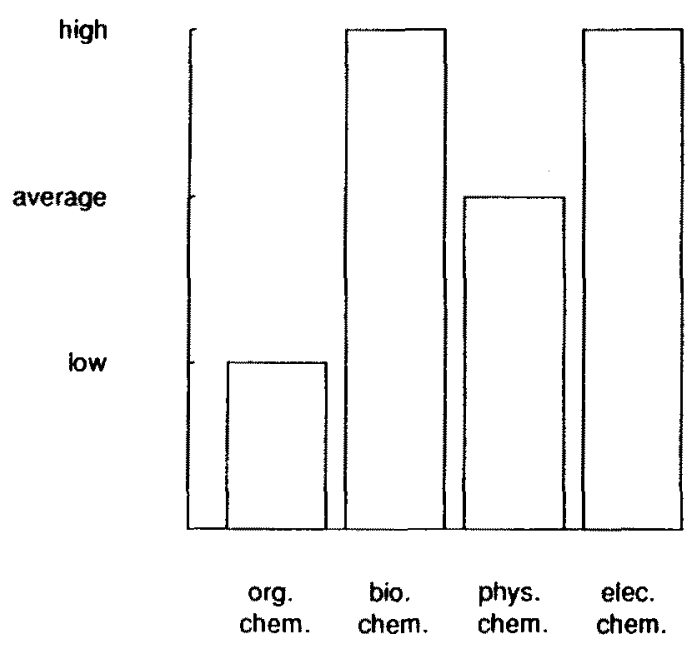

Student II

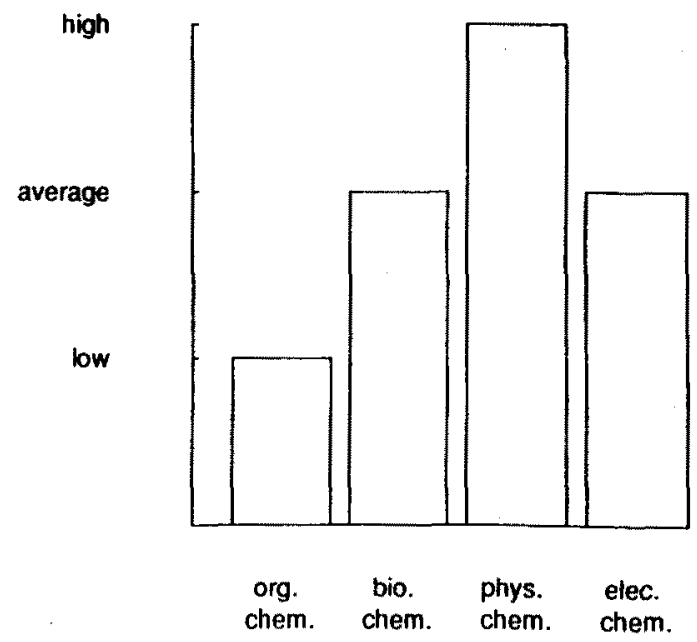

Figure 8. Graphical representations of pairs of score profiles. 
Table 11

Tabular Representation of a Pair of Achievement Profiles: Experiment 8

\begin{tabular}{lll}
\hline Chemistry subject & Student 1 & Student 2 \\
\hline Organic chemistry & Low & Low \\
Biochemistry & High & Average \\
Physical chemistry & Average & High \\
Electrochemistry & High & Average \\
\hline
\end{tabular}

difference in $\delta$. Hence the cohesiveness effect cannot be explained by baseline similarity.

This series of studies was motivated by the attempt to illuminate the finding that the weight of common components in relation to distinctive components is higher for verbal than for pictorial stimuli (Gati \& Tversky, 1984). Although Table 14 shows that baseline similarity cannot explain the effect of cohesiveness, it can perhaps explain the effect of modality. Because the verbal and the pictorial stimuli are very different, however, they cannot be compared directly as in Table 14 . Furthermore, as we noted earlier, $W$ is expected to covary with baseline similarity. The critical question, then, is whether stimulus modality (verbal or pictorial) contributes to $W$ over and above the effect of baseline similarity.

To answer this question, we reanalyzed all the withinsubject data of Gati and Tversky (1984) involving verbal comparisons (Studies 2, 3, 5, 6, 7) and pictorial comparisons (Studies, 8, 9, 10, 11). We computed $W$ separately for each additive component and for each subject and regressed these values against (a) the corresponding baseline similarity and (b) stimulus modality (verbal, pictorial). The simple correlation between $W$ and baseline similarity was -.34 , and the correlation between $W$ and modality was .30 . Baseline similarity alone accounted for $11.5 \%$ of the variance in $W$, and the inclusion of stimulus modality increased this value by $7.2 \%$, which represents a highly significant contribution $(p<$ $.001)$. The residual variance includes, besides error, individual differences and variations among stimuli. The difference between the verbal and the pictorial stimuli, therefore, had a significant effect on $W$ over and above the effect of baseline similarity. If the pictorial stimuli are indeed more cohesive than the verbal stimuli, then the effect of cohesiveness could help explain the modality effect.

Our findings raise a new question: Why is $W$ lower for cohesive stimuli than for less cohesive stimuli? One possible explanation of this effect invokes the hypothesis that $W$ is inversely related to the prevalence of the additive component.

Table 12

Summary Results for Experiment 8

(Within-Subject Analyses; $n=90$ )

\begin{tabular}{lcccc}
\hline Display & $C$ & $D$ & $\delta$ & $W$ \\
\hline Graphs & 2.25 & 3.25 & -1.00 & .39 \\
Lists & 2.51 & 3.32 & -0.82 & .42 \\
\hline
\end{tabular}

Note. $C=$ weight of a feature as a common component. $D=$ weight of a feature as a distinctive component. $\delta=C-D . W=C /(C+D)$.
An unusual avocation (e.g., skydiving, bird watching) is expected to yield higher $W$ than a more popular avocation (e.g., jogging, photography) because the sharing of a rare avocation is more informative than the sharing of a prevalent one. This account, however, does not explain the result of Experiments $2-7$, in which the same components were added to the cohesive and to the less cohesive stimuli.

One could argue, following Garner (1970), that the cohesive stimuli invoke a smaller set of possibilities than do the less cohesive stimuli because the constraints imposed on the relations among the components restrict the space of possible stimuli. As Garner put it, "Good patterns have few alternatives." As a consequence, the occurrence of a common component is less surprising and less informative for cohesive than for noncohesive stimuli. For example, if the set of possible snacks is perceived as much greater than the set of possible dinners, then the presence of a common dish will have greater impact in the comparison of snacks than in the comparison of dinners.

The most basic property of the contrast model (Tversky, 1977) is the representation of similarity in terms of common and distinctive features. Experimental research stimulated by this conception has uncovered several factors that control the relative weight of these components. Tversky and Gati (1978) showed that $W$ is larger in judgments of similarity than in judgments of dissimilarity, presumably because the former focus on shared features, whereas the latter focus on distinctive features. Gati and Tversky (1984) showed that the value of $W$ associated with a particular component, added to one or two subjects, is inversely related to the baseline similarity of the objects. This seems like a figure-ground effect: A few distinctive features stand out on the background of many common features, and a few common features stand out on the background of many distinctive features. Our experiments revealed an additional factor that controls $W$ : namely, the cohesiveness of the stimuli. Cohesive pictures and structured descriptions give rise to lower $W$ than do scrambled pictures and unstructured descriptions.

The use of similarity as an explanatory concept has been criticized by modern philosophers, notably Goodman (1972), on the grounds that it is context dependent, unstable, and even indeterminate. A pair of objects, it is argued, can be viewed as similar or dissimilar, depending on whether one attends to their common features or to their distinctive features. Our research, in which similarity was employed as a dependent, not an explanatory, variable, shows that the weighting of common and distinctive features is context dependent, but these variations are systematic rather than ran-

Table 13

Summary Results for Experiment 9

\begin{tabular}{lccccc}
\hline \multicolumn{1}{c}{ Condition } & $n$ & $C$ & $D$ & \multicolumn{1}{c}{$\delta$} & $W$ \\
\hline Drawings & 25 & 3.61 & 3.61 & 0.00 & .51 \\
Verbal descriptions & 22 & 2.38 & 3.46 & -0.98 & .36 \\
\hline
\end{tabular}

Note. $C=$ weight of a feature as a common component. $D=$ weight of a feature as a distinctive component. $\delta=C-D . W=C /(C+D)$. 
Table 14

Comparison of Baseline Similarity and $\delta$ for Cohesive (Co) and Noncohesive (NCo) Stimuli

\begin{tabular}{|c|c|c|c|c|c|c|c|c|c|}
\hline \multicolumn{2}{|c|}{ Experiment } & \multicolumn{2}{|c|}{ Manipulation } & \multicolumn{3}{|c|}{ Baseline similarity } & \multicolumn{3}{|c|}{$\delta$} \\
\hline Number & Stimuli & $\mathrm{Co}$ & $\mathrm{NCo}$ & $\mathrm{Co}$ & NCo & $p$ & $\mathrm{Co}$ & $\mathrm{NCo}$ & $p$ \\
\hline 2 & Scenes & $\begin{array}{l}\text { Horizontal } \\
\text { line }\end{array}$ & Vertical line & 6.49 & 7.16 & $n s$ & 1.42 & 2.65 & .05 \\
\hline 4 & Pictures & $\begin{array}{l}\text { Uniform } \\
\text { components }\end{array}$ & $\begin{array}{l}\text { Mixed } \\
\text { components }\end{array}$ & 7.29 & 8.11 & $n s$ & 0.30 & 2.75 & .05 \\
\hline 5 & Pictures & $\begin{array}{l}\text { Uniform } \\
\text { context }\end{array}$ & Mixed context & 7.08 & 7.67 & $n s$ & 0.41 & 3.19 & .05 \\
\hline 8 & Students & Graphs & Lists & 13.25 & 13.48 & $n s$ & -1.00 & -0.82 & $n s$ \\
\hline 9 & Figures & Drawings & Descriptions & 10.81 & 10.56 & $n s$ & 0.00 & -0.98 & $n s$ \\
\hline
\end{tabular}

dom; they reflect the nature of the task, the overall similarity of the stimuli, and the cohesiveness of their components.

\section{References}

Garner, W. R. (1970). Good patterns have few alternatives. American Scientist, 58, 34-42.

Gati, I., \& Tversky, A. (1984). Weighting common and distinctive features in perceptual and conceptual judgments. Cognitive Psychology, 16, 341-370.

Gati, 1., \& Tversky, A. (1987). Recall of common and distinctive features of verbal and pictorial stimuli. Memory \& Cognition, 15, 97-100.
Goodman, N. (1972). Seven strictures on similarity. In N. Goodman (Ed.), Problems and projects (pp. 437-446). New York: BobbsMerrill.

Tversky, A. (1977). Features of similarity. Pyschological Review, 84, 327-352.

Tversky, A., \& Gati, I. (1978). Studies of similarity. In E. Rosch \& B. Lloyd (Eds.), Cognition and categorization (pp. 79-98). Hillsdale, NJ: Erlbaum.

Received February 6, 1989

Revision received June 23, 1989

Accepted June 29, 1989

\section{Members of Underrepresented Groups: Reviewers for Journal Manuscripts Wanted}

If you are interested in reviewing manuscripts for APA journals, the APA Publications and Communications Board would like to invite your participation. Manuscript reviewers are vital to the publication process. As a reviewer, you would gain valuable experience in publishing. The P\&C Board is particularly interested in encouraging members of underrepresented groups to participate in this process.

If you are interested in reviewing manuscripts, please write to Leslie Cameron at the address below. Please note the following important points:

- To be selected as a reviewer, you must have published articles in peer-reviewed journals. The experience of publishing provides a reviewer with the basis for preparing a thorough, objective evaluative review.

- To select the appropriate reviewers for each manuscript, the editor needs detailed information. Please include with your letter your vita. In your letter, please identify which APA journal you are interested in and describe your area of expertise. Be as specific as possible. For example, "social psychology" is not sufficient-you would need to specify "social cognition" or "attitude change" as well.

- Reviewing a manuscript takes time. If you are selected to review a manuscript, be prepared to invest the necessary time to evaluate the manuscript thoroughly.

Write to Leslie Cameron, Journals Office, APA, 1400 North Uhle Street, Arlington, Virginia 22201. 